\title{
Social determinants of common metabolic risk factors (high blood pressure, high blood sugar, high body mass index and high waist-hip ratio) of major non- communicable diseases in South Asia region: a systematic review protocol
}

Sudesh Raj Sharma ${ }^{1,3^{*}}$, Shiva Raj Mishra ${ }^{1 *}$, Kusum Wagle ${ }^{1,2}$, Rachel Page ${ }^{3}$, Anna Matheson $^{3}$, Danielle Lambrick ${ }^{4}$, James Faulkner ${ }^{5}$, David Lounsbury ${ }^{6}$ and Abhinav Vaidya ${ }^{7}$

\begin{abstract}
Background: Prevalence of non-communicable diseases has been increasing at a greater pace in developing countries and, in particular, the South Asia region. Various behavioral, social and environmental factors present in this region perpetuate common metabolic risk factors of non-communicable diseases. This study will identify social determinants of common metabolic risk factors of major non-communicable diseases in the context of the South Asian region and map their causal pathway.

Methods: A systematic review of selected articles will be carried out following Cochrane guidelines. Review will be guided by Social Determinants of Health Framework developed by the World Health Organization to extract social determinants of metabolic risk factors of non-communicable diseases from studies. A distinct search strategy will be applied using key words to screen relevant studies from online databases. Primary and grey literature published from the year 2000 to 2016 and studies with discussion on proximal and distal determinants of non-communicable risk factors among adults of the South Asia region will be selected. They will be further checked for quality, and a matrix illustrating contents of selected articles will be developed. Thematic content analysis will be done to trace social determinants and their interaction with metabolic risk factors. Findings will be illustrated in causal loop diagrams with social determinants of risk factors along with their interaction (feedback mechanism).

Discussion: The review will describe the interplay of social determinants of common NCD metabolic risk factors in the form of causal loop diagram. Findings will be structured in two parts: the first part will explain the linkage between proximal determinants with the metabolic risk factors and the second part will describe the linkage among the risk factors, proximal determinants and distal determinants. Evidences across different regions will be discussed to compare and validate and/or contrast the findings. Possible bias and limitations of this study will also be discussed.
\end{abstract}

Systematic review registration: PROSPERO CRD42017067212

Keywords: NCDs, Risk factors, Social determinants, South Asia

\footnotetext{
* Correspondence: yoursudesh@gmail.com; shivarajmishra@gmail.com

${ }^{1}$ Nepal Development Society, Kathmandu, Nepal

Full list of author information is available at the end of the article
} 


\section{Background}

Global burden of non-communicable diseases (NCDs) has been increasing, and the South Asia region in particular is showing a rapid increase [1]. In this region, nearly half of the disease burden and two-thirds of the total deaths are caused by NCDs [2]. In addition to mortality, disability due to NCDs is posing a serious economic burden due to reduced productivity and poverty among affected people who need long-term care [3, 4]. Cardiovascular diseases, respiratory diseases, cancers and diabetes are four major diseases causing deaths and creating burden in this region [1]. Common risk factors for them are poor dietary habits, tobacco use and inadequate physical activity and metabolic risk factors such as high blood pressure, high blood sugar, high Body mass index (BMI) and high waist-hip ratio (WHR) [5-8] [Additional file 1: Appendix 1]. NCDs have a complex causal mechanism characterized by having multiple etiologies and factors [9]. Factors causing NCDs are influenced by an individual's behavior along with its interaction with the external socio-economic environment, on the top of the genealogical predisposition that people are born with $[10,11]$. Thus, changing individuals' behavior is not adequate in itself without addressing underlying the social, economic, environmental and cultural factors collectively known as social determinants of health (SDH) [12]. A SDH framework has been proposed by the World Health Organization to elucidate these complexities [13]. This framework is based on Social Production of Disease Theory and integrates the best available evidences on social determinants of health. The framework is increasingly being utilized to understand and address multifactorial and complex causation of health problems like NCDs $[12,14,15]$. In the framework, the proximal determinants include behavioral and psychosocial factors, material circumstances, community capital and cohesion and health system factors whereas distal determinants include social positioning/ class and socio-economic context (Fig. 1).

The lack of progress in addressing these social determinants of complex health problems indicates that our existing approaches are inadequate and may need help from an alternative science. Complexity sciences or system sciences hold the key to addressing these complex public health problems by improving our existing public health approaches [16, 17]. System sciences methods and tools are well placed for understanding the dynamic structure and emergent behavior of a social system leading to complex health problems. Frameworks like SDH could be further improved and contextualized through identifying gaps in practice, and enable better design of effective interventions if a complex systems approach is applied [18].

There is a need to understand social determinants of common metabolic risk factors of major NCDs for systematic action in the South Asia region using system science approach and tool. However, there is a scarcity of such comprehensive systematic reviews on social determinants of common metabolic risk factors in the South Asia region. This systematic review will first identify the common social determinants of major NCDs and then map the causal pathways through which those determinants interact to perpetuate common metabolic risk factors (high blood pressure, high blood sugar, high BMI and high WHR) among the adult population in South Asia.

\section{Methods}

The systematic review will be conducted following the Cochrane guidelines [19]. A Preferred Reporting Items for Systematic Reviews and Meta-Analyses for Protocols

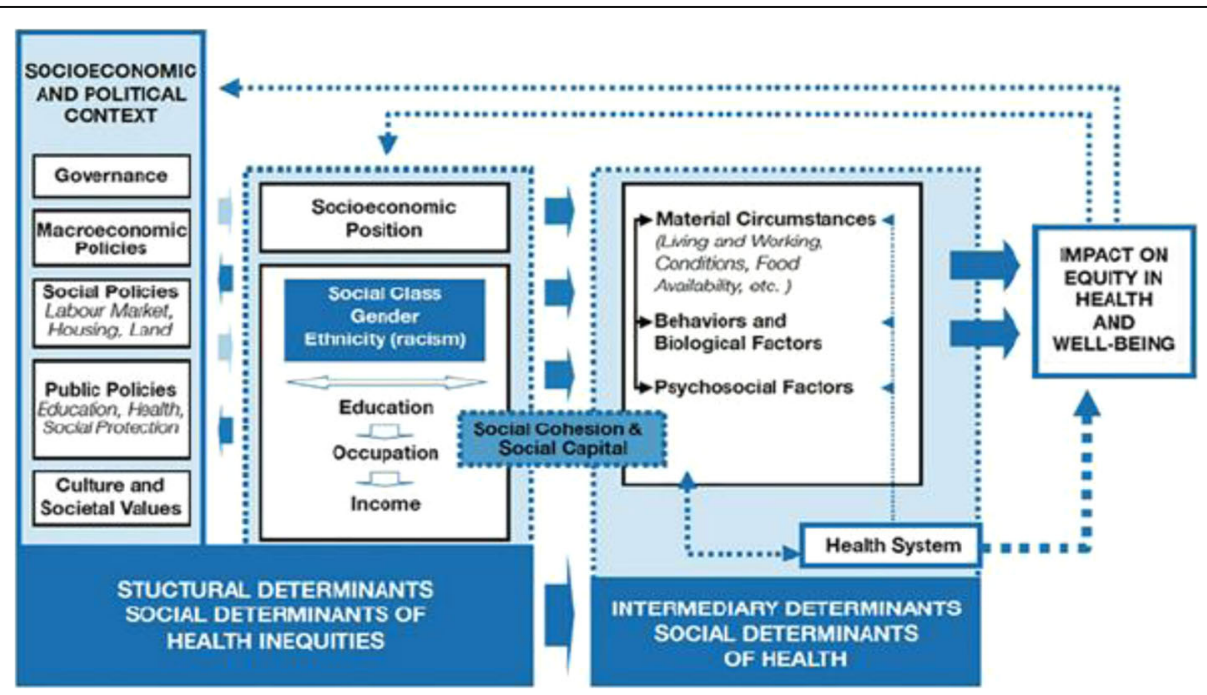

Fig. 1 Social determinants of health framework proposed by the WHO Commission on SDH (link, http://www.who.int/sdhconference/resources/ ConceptualframeworkforactiononSDH_eng.pdf) 
(PRISMA-P) checklist has been completed to ensure that the protocol is robust [20] [Additional file 2: Appendix II]. We will use thematic content analysis to identify social determinants of NCD risk factors and map the causal pathways leading to major NCDs in the South Asia region using the WHO framework described earlier.

\section{Search strategy}

We will search for relevant published articles and grey literature through PubMed, Embase, Web of Science and ProQuest Central using sequence of key words listed in Table 1. A detailed search strategy using Boolean operators and contractions for key search terms will be developed in consultation with a librarian at the Massey University library in Wellington.

\section{Inclusion criteria}

Inclusion criteria for selection of studies and grey literature will be as follows: (a) primary research and grey literature published between 2000 and 2016, (b) studies among an adult population aged 20-65 from South Asian region (Nepal, India, Bangladesh, Pakistan, Sri Lanka, Bhutan, Maldives, Afghanistan) and (c) studies and literature that have generated empirical evidences on proximal and/or distal determinants of the selected NCD risk factors.

Both published and unpublished, grey and peer reviewed, and qualitative and quantitative studies will be included. Any study outside of the South Asia region and not mentioning at least one of the proximal and/or one of the distal determinants from the SDH framework will be excluded [List in Additional file 3: Appendix III].

\section{Screening procedures}

Studies will be primarily screened on the basis of inclusion criteria. Two independent reviewers (SRS and KW) will review the title and abstract checking all the inclusion criteria. We will use Covidence (https://www.covidence.org/), a Cochrane Technology Platform tool to systematize the process. The secondary screening will involve full-text review, review of methods including selected cutoff values for metabolic risk factors [Additional file 1: Appendix I] and review of findings and discussion section for causal pathway analysis on social determinants of NCD risk factors. The team will exclude the duplicated articles and then finalize the list for systematic review.

\section{Quality assessment of research papers}

The selected articles will be assessed for their quality using QUALSYST tool [21]. There are many tools (for example, Effective Public Health Practice Project-EPHPP and National Institute of Health Quality Assessment Tool for quantitative studies; Popay et al. criteria and Mays et al. criteria for qualitative studies) to assess the quality of research papers [22-25], but limited tools/criteria have incorporated checklists for both qualitative and quantitative research designs. QUALYSYST tool has been validated for quality assessment of both qualitative and quantitative studies for systematic review. Further, the tool is easy to use and draws upon existing tools to address the issue of simultaneous assessment of the quality of studies which are diverse in nature. Two independent researchers (SRS and KW) will assess the quality of the studies and exclude papers with low quality score (Interrater agreement score of $<0.55)$. The final list will be agreed upon by both researchers (SRS and KW) for data extraction and analysis.

\section{Data extraction and management}

Data will be extracted from the final list of articles by the research team in an illustrative matrix as shown in Table 2.

\section{Data analysis including mapping of causal pathways}

Thematic analysis will be performed to identify patterns/ themes across the extracted data/codes and stream out common pathways from determinants to risk factors of NCD simultaneously to data extraction. The team will meet regularly to check the consistency in data extraction and analysis and discuss issues, if any.

The identified causal linkages/pathways will be further consolidated as per the SDH framework and mapped in the form of causal loop diagram (CLD). CLD is a system dynamic mapping tool to express the dynamic causal

Table 1 Key words for searching articles for the review

\begin{tabular}{lll}
\hline Key words & Other variations of key words & \\
\hline $\begin{array}{l}\text { NCDs metabolic risk factors plus } \\
\text { proximal determinants plus distal } \\
\text { determinants plus geographic location }\end{array}$ & NCDs metabolic risk factors & $\begin{array}{l}\text { High blood pressure, hypertension, high blood glucose, } \\
\text { hyperglycemia, diabetes, high BMl, obesity, overweight, } \\
\text { waist circumference, waist-hip ratio } \\
\text { Determinants, risk, diet, salt intake, tobacco, smoking, } \\
\text { alcohol, physical inactivity, lifestyle }\end{array}$ \\
& Proximal determinants & $\begin{array}{l}\text { Social determinants, education, income, gender, race, caste, } \\
\text { ethnicity, socio-economic status, wealth index, income }\end{array}$ \\
& Distal determinants & $\begin{array}{l}\text { Nepal, India, Bangladesh, Pakistan, Sri Lanka, Bhutan, Maldives, } \\
\text { Afghanistan }\end{array}$ \\
\hline
\end{tabular}


Table 2 Example of illustrative matrix of selected articles

\begin{tabular}{|c|c|c|c|c|c|c|c|}
\hline $\begin{array}{l}\text { Literature type and } \\
\text { year of publication }\end{array}$ & $\begin{array}{l}\text { Research } \\
\text { type }\end{array}$ & $\begin{array}{l}\text { Study objective/ } \\
\text { method }\end{array}$ & $\begin{array}{l}\text { Study location } \\
\text { and sample size }\end{array}$ & $\begin{array}{l}\text { Metabolic } \\
\text { risk factor }\end{array}$ & $\begin{array}{l}\text { Proximal } \\
\text { determinants }\end{array}$ & $\begin{array}{l}\text { Distal } \\
\text { determinants }\end{array}$ & $\begin{array}{l}\text { Causal linkage } \\
\text { thematic analysis }\end{array}$ \\
\hline $\begin{array}{l}\text { Primary article or } \\
\text { grey literature as } \\
\text { searched in three } \\
\text { databases/year } \\
\text { of publication } \\
\text { as mentioned }\end{array}$ & $\begin{array}{l}\text { Quantitative or } \\
\text { qualitative or } \\
\text { mixed-method } \\
\text { research }\end{array}$ & $\begin{array}{l}\text { Describe in } \\
\text { detail about the } \\
\text { study objective } \\
\text { and methods }\end{array}$ & $\begin{array}{l}\text { Country and } \\
\text { region where the } \\
\text { study was taken } \\
\text { and sample size }\end{array}$ & $\begin{array}{l}\text { Metabolic risk } \\
\text { factor under } \\
\text { study }\end{array}$ & $\begin{array}{l}\text { See } \\
\text { Additional file 3: } \\
\text { Appendix III }\end{array}$ & $\begin{array}{l}\text { See } \\
\text { Additional file 3: } \\
\text { Appendix III }\end{array}$ & $\begin{array}{l}\text { Coding and } \\
\text { thematic analysis } \\
\text { about the possible } \\
\text { causal linkage among } \\
\text { determinants based } \\
\text { on the results and } \\
\text { discussion of the } \\
\text { article }\end{array}$ \\
\hline
\end{tabular}

relationships operating among variables giving rise to a specific situation. It is based on control and feedback theory and mainly driven by the endogenous behavior of the variables in generating a specific problem and how the problem, in return, is providing feedback to the determinants. The CLD from the review will show the linkages between social determinants of common NCD risk factors, and how these determinants reinforce or balance each other. The strategy to construct CLD in this review will be based on Business Dynamics, a system science resource [26].

An example of casual loop diagram depicting the issue of Junk Food control is presented below. There are two loops in the diagram, one is balancing loop indicated by $B$ in the centre of the loop and the other is the reinforcing loop indicated by $R$ in the centre, see Fig. 2 .

The balancing loop is a goal-seeking loop which tries to bring down the availability and affordability of the junk food however is unable to do so due to system delays (indicated by // sign in the arrow). The positive linkage (+ sign in the arrowhead) between Junk Food availability, accessiblity and affordability and Health effects indicates that the universal availability and affordability of junk food is giving rise to health effects. The increasing health effects are in-turn providing pressure to the authorities to develop regulatory policies which are delayed. These regulatory policies again have delayed implementation and are often in action when the problem has already grown out of proportion with other unintended consequences. On the left-hand side of the figure, the reinforcing loop indicates how the junk food companies utilize their profits to bolster marketing of their products and influence policy for delayed implementation of regulatory policies inorder to maintain their sales and profit. The causal loop diagram will be built using the "Vensim", a freely available software program for developing system models [27].

\section{Discussion}

The findings will describe how various social determinants interact and influence each other in the South Asian population brewing and propagating an epidemic of common metabolic risk factors of NCD. Our review will depict such interactions within and outside of the health and social systems using a causal loop diagram based on qualitative analysis of selected articles. The review will be structured in two parts. The first part would explain the linkage between proximal determinants with the metabolic risk factors. The second part will describe the linkage among distal determinants, proximal determinants and the metabolic risk factors of major NCDs in this region. Regional and country wise comparison will be done to validate and/or contrast the findings. Causal loop diagrams of social determinants of common metabolic risk factors of NCDs will provide insights on how the social determinants are linked with each other in

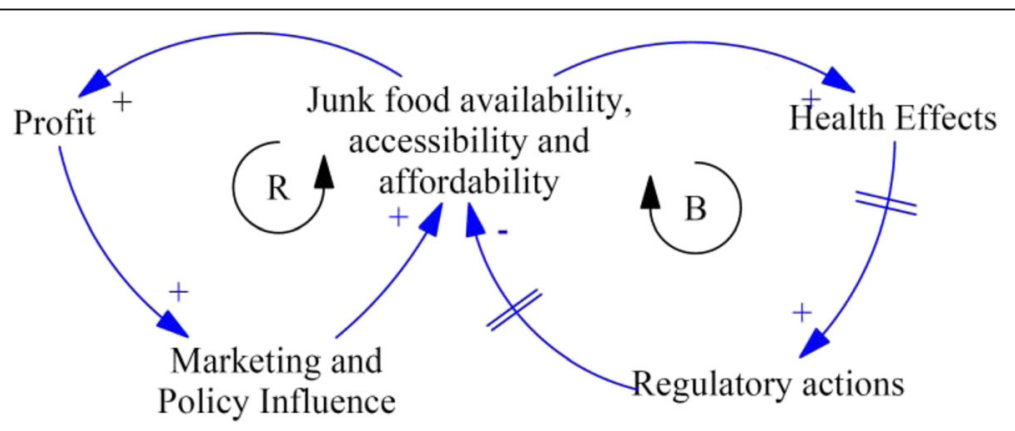

Fig. 2 Example of causal loop diagram of an issue of Junk Food control 
perpetuating NCD problem for the South Asia region context. The review will have a much needed policy and programmatic implications in the current situation of increasing burden of NCD in South Asia. However, this review will be limited to articles written in English language and identified through four online databases.

\section{Additional files}

Additional file 1: Appendix l: Cutoff values of metabolic risk factors of NCDs (DOCX $15 \mathrm{~kb}$ )

Additional file 2: Appendix II: PRISMA-P checklist for the systematic review (DOCX $21 \mathrm{~kb}$ )

Additional file 3: Appendix III: List of proximal and distal determinants (based on SDH framework) (DOCX $16 \mathrm{~kb}$ )

\section{Abbreviations}

BMI: Body mass index; CLD: Causal loop diagram; NCDs: Non-communicable diseases; PRISMA-P: Preferred Reporting Items for Systematic Reviews and Meta-Analyses for Protocols; SDH: Social determinants of health; WHO: World Health Organization; WHR: Waist-hip ratio

\section{Acknowledgements}

We would like to acknowledge the support provided by the Nepal Development Society and Massey University Library.

\section{Funding}

The authors have received no funding for carrying out this review.

\section{Availability of data and materials}

Not applicable.

\section{Authors' contributions}

SRS conceived and drafted the initial manuscript. SRM, RP, AM, JF, KW, DL and AV all critically reviewed and revised the initial manuscript. SRS and KW prepared the final manuscript. All authors read and approved the final manuscript.

\section{Authors' information}

Sudesh Raj Sharma (MPH, MHCom.) is a public health professional from Nepal and is currently studying for a PhD at Massey University. Shiva Raj Mishra (MPH) is a cardiovascular epidemiologist with skills in global health, health systems and policy. Kusum Wagle (MPH) is a researcher and is currently working at CREHPA, a research institute in Nepal. Professor Rache Page (PhD) is Head of School of Health Sciences at Massey University with over 20 years research experience in the area of metabolism and disease process. Anna Matheson (PhD) is a public health teacher and researcher at Massey University with expertise in the social determinants of health and equity, complex systems and social intervention. Danielle Lambrick (PhD) is a Lecturer in Life Sciences within the Faculty of Health Sciences, University of Southampton. James Faulkner (PhD) is a Reader in Exercise Physiology at the University of Winchester, UK. David Lounsbury (PhD) is an Assistant Professor of Epidemiology and Population Health at the Albert Einstein College of Medicine, Bronx, NY, USA. Abhinav Vaidya (MD, PhD) is an Associate Professor at Department of Community Medicine, Kathmandu Medical College, Kathmandu, Nepal. SRS and SRM are members of Nepal Development Society (NEDS).

\section{Ethics approval and consent to participate}

Not applicable.

\section{Consent for publication}

All the authors have consent for submission and publication.

\section{Competing interests}

We declare no conflict of interest.

\section{Publisher's Note}

Springer Nature remains neutral with regard to jurisdictional claims in published maps and institutional affiliations.

\section{Author details}

${ }^{1}$ Nepal Development Society, Kathmandu, Nepal. ${ }^{2}$ James P. Grant School of Public Health, BRAC University, Dhaka, Bangladesh. ${ }^{3}$ College of Health, Massey University, Wellington, New Zealand. ${ }^{4}$ University of Southampton, Southampton, UK. ${ }^{5}$ University of Winchester, Winchester, UK. ${ }^{6}$ Albert Einstein College of Medicine, Bronx, NY 10461, USA. ${ }^{7}$ Kathmandu Medical College, Kathmandu, Nepal.

Received: 22 May 2017 Accepted: 29 August 2017

Published online: 07 September 2017

\section{References}

1. Siegel KR, Patel SA, Ali MK. Non-communicable diseases in South Asia: contemporary perspectives. Br Med Bull. 2014;111(1):31-44.

2. Ghaffar A, Reddy KS, Singhi M. Burden of non-communicable diseases in South Asia. BMJ. 2004;328(7443):807.

3. Lazzari A, de Waure C, Azzopardi-Muscat N. A Systematic Review of Key Issues in Public Health. Switzerland: Springer International Publishing; 2015.

4. Dans A, Ng N, Varghese C, Tai ES, Firestone R, Bonita R. The rise of chronic non-communicable diseases in southeast Asia: time for action. Lancet. 2011 377(9766):680-9.

5. Afshin A, Micha R, Khatibzadeh S, Fahimi S, Shi P, Powles J, Singh G, Yakoob MY, Abdollahi M, Al-Hooti S. The impact of dietary habits and metabolic risk factors on cardiovascular and diabetes mortality in countries of the Middle East and North Africa in 2010: a comparative risk assessment analysis. BMJ Open. 2015;5(5):e006385

6. Ezzati M, Riboli E. Behavioral and dietary risk factors for noncommunicable diseases. N Engl J Med. 2013;369(10):954-64

7. Saeed KMI. Prevalence of risk factors for non-communicable diseases in the adult population of urban areas in Kabul City, Afghanistan. Central Asian Journal of Global Health. 2014;2(2). https://cajgh.pitt.edu/ojs/index.php/ cajgh/article/view/69/144.

8. World Health Organization: Global Health Observatory (GHO) data, risk factors. 2015.

9. Centers for Disease Control Prevention: Overview of non-communicable diseases and related risk factors. 2015

10. Alamian A, Paradis $\mathrm{G}$. Individual and social determinants of multiple chronic disease behavioral risk factors among youth. BMC Public Health. 2012;12(1): 224.

11. Blas E, Kurup AS. Equity, social determinants and public health programmes. Switzerland: World Health Organization; 2010.

12. Bell R, Lutz B. Disscussion paper: addressing the social determinants of noncommunicable diseases. USA: UNDP; 2013.

13. Solar O, Irwin A. A conceptual framework for action on the social determinants of health, Social Determinants of Health Discussion Paper 2 (Policy and Practice). Geneva: World Health Organization; 2010.

14. Bahadori M, Sanaeinasab H, Ghanei M, Mehrabi Tavana A, Ravangard R, Karamali $\mathrm{M}$. The social determinants of health $(\mathrm{SDH})$ in Iran: a systematic review article. Iran J Public Health. 2015;44(6):728-41.

15. Horton K, Friel S, de Leeuw E, McKeone C, Bolam B. Fair foundations for health equity. Health Promot Int. 2015;30(suppl_2):ii2-ii7.

16. Green LW. Public health asks of systems science: to advance our evidencebased practice, can you help us get more practice-based evidence? Am J Public Health. 2006;96(3):406-9.

17. Tremblay M-C, Richard L. Complexity: a potential paradigm for a health promotion discipline. Health Promot Int. 2014;29(2):378-88.

18. Matheson A. Reducing social inequalities in obesity: complexity and power relationships. J Public Health. 2016;38(4):826-9.

19. Higgins JP, Green S. Cochrane handbook for systematic reviews of interventions. England: John Wiley \& Sons. 2011;4.

20. Moher D, Shamseer L, Clarke M, Ghersi D, Liberati A, Petticrew M, Shekelle P, Stewart LA. Preferred reporting items for systematic review and metaanalysis protocols (PRISMA-P) 2015 statement. Syst Rev. 2015:4(1):1.

21. Kmet LM, Lee RC, Cook LS. Standard quality assessment criteria for evaluating primary research papers from a variety of fields. Edmonton: Alberta Heritage Foundation for Medical Research; 2004. 
22. Thomas B, Ciliska D, Dobbins M, Micucci S. A process for systematically reviewing the literature: providing the research evidence for public health nursing interventions. Worldviews on Evidence-Based Nursing. 2004;1(3): 176-84.

23. Health Nlo. Quality assessment tool for observational cohort and crosssectional studies. National Heart, Lung, and Blood Institute Available from: www nhlbi nih gov/health-pro/guidelines/in-develop/cardiovascular-riskreduction/tools/cohort [Accessed November 5, 2015] 2014.

24. Mays N, Pope C. Qualitative research in health care: assessing quality in qualitative research. BMJ. 2000;320(7226):50.

25. Popay J, Rogers A, Williams G. Rationale and standards for the systematic review of qualitative literature in health services research. Qual Health Res. 1998;8(3):341-51.

26. Sterman JD. Business dynamics: systems thinking and modeling for a complex world. Boston: Irwin/McGraw-Hill; 2000.

27. Vensim Home [http://vensim.com/].

Submit your next manuscript to BioMed Central and we will help you at every step:

- We accept pre-submission inquiries

- Our selector tool helps you to find the most relevant journal

- We provide round the clock customer support

- Convenient online submission

- Thorough peer review

- Inclusion in PubMed and all major indexing services

- Maximum visibility for your research

Submit your manuscript at www.biomedcentral.com/submit
C) Biomed Central 